Paul Klein

Department of Economics

University of Western Ontario

\title{
Parameterized expectations
}

See also Marcet and Marshall (1994) and Lorenzoni and Marcet (1999).

\section{The method}

We start with a set of equilibrium conditions on the following form.

$$
\left\{\begin{aligned}
f\left(x_{t}, d_{t}, e_{t}\right) & =0 \\
x_{t+1} & =g\left(x_{t}, d_{t}, z_{t+1}\right) .
\end{aligned}\right.
$$

where

$$
e_{t}=\mathrm{E}_{t}\left[h\left(d_{t+1}, x_{t+1}\right)\right]
$$

and $z_{t}$ is i.i.d.

Under mild regularity conditions, there exists a function $\xi$ such that

$$
e_{t}=\xi\left(x_{t}\right)
$$

Suppose, for a moment, that we know the function $\xi$. Then the decision function $d$ solves the following trivial functional equation.

$$
f(x, d(x), \xi(x))=0 .
$$

The PEA algorithm proceeds, as the name suggests, by parameterizing the expectations.

$$
\xi(x) \approx \psi(x ; \theta)
$$


If we know $\theta$ then we are in a position to simulate time series $\left\{x_{t}\right\}$ and $\left\{d_{t}\right\}$. Once that is done, we can update $\theta$ so as to minimize, with respect to $\widetilde{\theta}$ the sum of squared deviations

$$
\sum_{t=0}^{T-1}\left(\psi\left(x_{t} ; \widetilde{\theta}\right)-h\left(x_{t+1}, d_{t+1}\right)\right)^{2}
$$

where the square is interpreted elementwise.

\section{Non-linear regression}

Define

$$
y_{t}=h\left(x_{t+1}, d_{t+1}\right) .
$$

You already know what to do if $\psi$ is a linear function of $\theta$, i.e. $\psi(x ; \theta)=x^{\prime} \theta$. Then all you do is choose $\theta$ so as to satisfy the orthogonality condition

$$
\sum_{t=0}^{T} x_{t}\left(y_{t}-x_{t}^{\prime} \theta\right)=0 .
$$

To do this, you define

$$
A=\sum_{t=0}^{T} x_{t} x_{t}^{\prime}
$$

and

$$
b=\sum_{t=0}^{T} x_{t} y_{t}^{\prime} .
$$

Then solve the linear system of equations

$$
A \theta=b .
$$

But what if $\theta$ is non-linear? Then you want to satisfy the first order condition for minimizing (1). The orthogonality condition then looks just as before, except that $x_{t}$ is replaced by

$$
\widetilde{x}_{t}=\frac{\partial \psi\left(x_{t}, \theta\right)}{\partial \theta} .
$$


The only problem with this is that the derivative itself depends on $\theta$, so iteration is required. As an initial guess, you obviously use the previous value of $\theta$.

\section{Homotopy}

Having a good initial guess is crucial. To make sure you have a good one, it makes sense to begin with a model whose solution you know analytically.

\section{References}

Lorenzoni, G. and A. Marcet (1999). The parameterized expectations approach: Some practical issues. In R. Marimon and A. Scott (Eds.), Computational Methods for the Study of Dynamic Economies, Chapter 7. Oxford University Press.

Marcet, A. and D. A. Marshall (1994). Solving nonlinear rational expectations models by parameterized expectations: convergence to stationary solutions. Working Paper Series, Macroeconomic Issues 94-20, Federal Reserve Bank of Chicago. 